\title{
Intraoperative fluorescence molecular imaging accelerates the coming of precision surgery in China
}

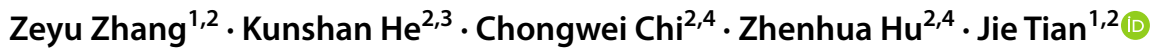

Received: 1 October 2021 / Accepted: 13 February 2022 / Published online: 1 March 2022

(c) The Author(s) 2022

\begin{abstract}
Purpose China has the largest cancer population globally. Surgery is the main choice for most solid cancer patients. Intraoperative fluorescence molecular imaging (FMI) has shown its great potential in assisting surgeons in achieving precise resection. We summarized the typical applications of intraoperative FMI and several new trends to promote the development of precision surgery.

Methods The academic database and NIH clinical trial platform were systematically evaluated. We focused on the clinical application of intraoperative FMI in China. Special emphasis was placed on a series of typical studies with new technologies or high-level evidence. The emerging strategy of combining FMI with other modalities was also discussed.

Results The clinical applications of clinically approved indocyanine green (ICG), methylene blue (MB), or fluorescein are on the rise in different surgical departments. Intraoperative FMI has achieved precise lesion detection, sentinel lymph node mapping, and lymphangiography for many cancers. Nerve imaging is also exploring to reduce iatrogenic injuries. Through different administration routes, these fluorescent imaging agents provided encouraging results in surgical navigation. Meanwhile, designing new cancer-specific fluorescent tracers is expected to be a promising trend to further improve the surgical outcome.

Conclusions Intraoperative FMI is in a rapid development in China. In-depth understanding of cancer-related molecular mechanisms is necessary to achieve precision surgery. Molecular-targeted fluorescent agents and multi-modal imaging techniques might play crucial roles in the era of precision surgery.
\end{abstract}

Keywords Molecular imaging · Precision surgery $\cdot$ Cancer surgery $\cdot$ Image-guided surgery $\cdot$ Molecular-targeted agents · Clinical translation

This article is part of the Topical Collection on Technology

Zeyu Zhang and Kunshan He contributed equally

Chongwei Chi

chongwei.chi@ia.ac.cn

$\triangle$ Zhenhua $\mathrm{Hu}$ zhenhua.hu@ia.ac.cn

$\bowtie$ Jie Tian

tian@ieee.org

1 Beijing Advanced Innovation Center for Big Data-Based Precision Medicine, School of Engineering Medicine, Beihang University, Beijing, China

2 CAS Key Laboratory of Molecular Imaging, Beijing Key Laboratory of Molecular Imaging, The State Key Laboratory of Management and Control for Complex Systems, Institute of Automation, Chinese Academy of Sciences, Beijing, China

3 State Key Laboratory of Computer Science and Beijing Key Lab of Human-Computer Interaction, Institute of Software, Chinese Academy of Sciences, Beijing, China

4 University of Chinese Academy of Sciences, Beijing, China 


\section{Introduction}

Surgery plays a vital role against the rising threat of cancer [1]. The expected goal of cancer surgery is to completely remove the cancer cells with minimum functional impairment in the least possible time [2]. To achieve this goal, high surgical precision is hugely required. Yet in the current stage, visual and tactile inspections are still the main ways for surgical decision making. These inspections are obviously experience-dependent and subjective which consequently restrain the surgical outcomes [2-4]. How to raise the surgical precision has become an important topic.

In the past centuries, various techniques have been developed to arm surgeons and the operation room. Computed tomography (CT), magnetic resonance imaging (MRI), ultrasound, and nuclear medicine imaging have become common tools for preoperative diagnosis [5]. These non-invasive imaging techniques can considerably help surgeons for surgical planning and treatment improvement $[6,7]$. Nevertheless, some problems regarding surgical guidance and decision making remain unsolved. The widely applied CT and MRI have limited sensitivity and accuracy to discover subtle lesions, and are hard to provide intraoperative real-time guidance. The practicability of positron emission tomography (PET) is also limited for surgical guidance, even though PET works well to detect small lesions and metastases [5]. Besides, intraoperative CT or PET may cause the concern of radioactive safety. Ultrasonography shows a strong practicability in both diagnosis and the surgical treatment. However, its quality is dependent on the examiner's experience and the superficial lesions (like the residues) might be occult in ultrasonography. Comprehensively speaking, an ideal surgical guidance technique would be better to have following qualities: high sensitivity, favorable resolution, adjustable vision, fast imaging speed, and non-radiative.

After several decades of evolution, the fluorescence molecular imaging (FMI) has now become an attractive modality to handle surgical problems regarding cancer [8]. With administration of fluorescent agents, FMI helps surgeons to make surgical decisions in a manner of real-time visualization (Fig. 1). Compared with the abovementioned CT, MRI, PET, or ultrasonography, FMI can reach picomolar sensitivity and micrometer spatial resolution and could acquire hundreds of images per second. The fluorescence images are intuitive to observers, which is beneficial to reduce the cost of operative training. Moreover, the dainty FMI imagers can be easily utilized in hands and transferred between different departments [4]. Thanks to these advantages, FMI shows a great potential in various surgical applications. From the 2000s, the FMI-guided surgery has been verified safe and effective in many applications [3,9]. An increasing number of surgeons are getting familiar with this promising technique. So far, a series of intraoperative FMI devices have been registered for clinical use in the fastgrowing market, which can serve endoscopy, laparoscopic and open surgery.

Imaging agent as another key element, its usage directly influences the application and the efficacy of FMI. Widely different from the device market, only few fluorescent imaging agents own the clinical approval up to now, including fluorescein (FS), methylene blue (MB), indocyanine green (ICG), and 5-aminolevulinic acid (5-ALA) [10].

Among them, ICG becomes the first choice in most clinical surgeries. The near-infrared (NIR) fluorescence
Fig. 1 The crucial steps of the clinical translation of novel intraoperative FMI techniques

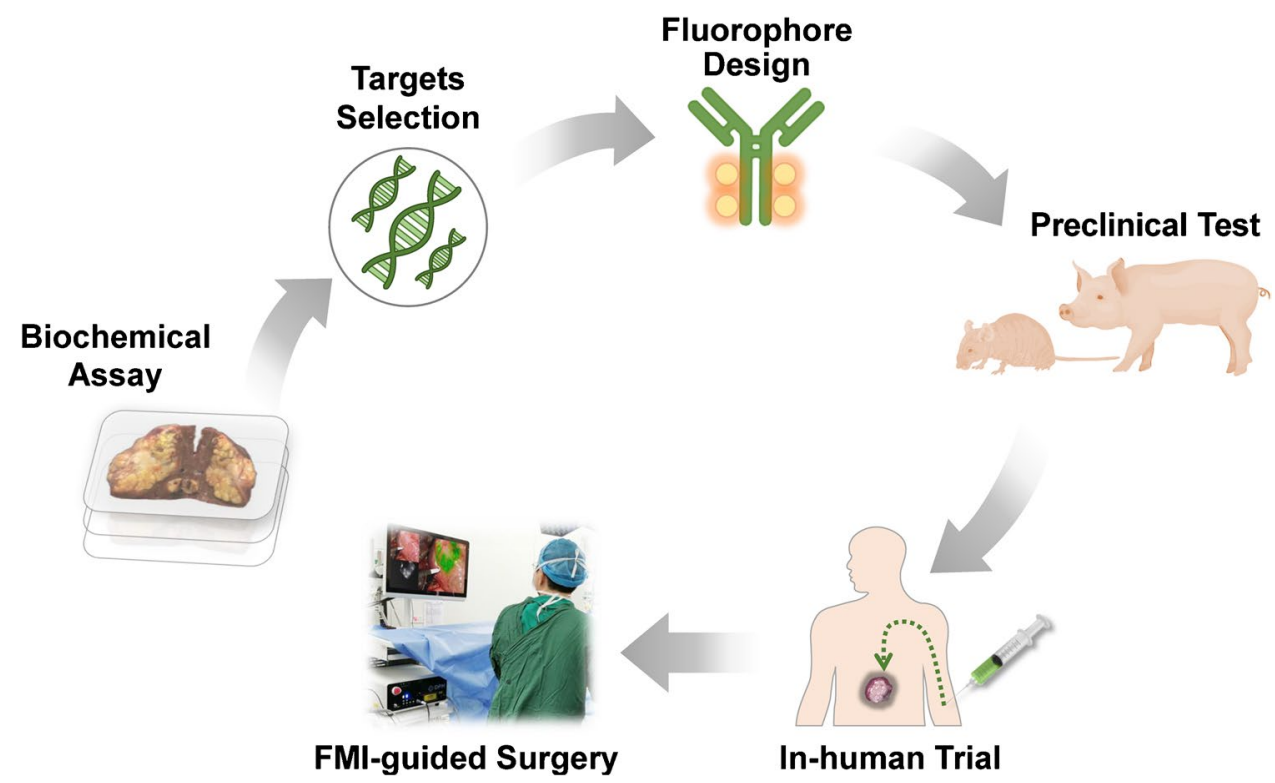


generated by ICG has strong imaging contrast, good resolution, and ideal penetration in vivo. However, these 4 approved fluorescent imaging agents do not have the capability of targeting cancer cells. Non-ideal sensitivity or falsepositive has been widely reported in diverse trials. Current achievements are still far from the expected high-precision cancer surgery. Recent progresses originated from Europe and the USA bring a new trend to develop molecular targeted fluorescent imaging agents [11]. A new folate receptor-alpha targeting agent "OTL38" has been approved by FDA in November 2021 [12]. This would be a milestone for marching in the era of precision surgery.

As for China, the world's largest cancer population leads to an increasing requirement of precision surgery. Because of late diagnosis, more resectable cancer cases may have a higher stage in China. Trials involving these patients could help better understand how much prognosis could be improved by FMI-guided surgery. China also has special molecular profiles in many cancers. For instance, approximately $61 \%$ lung adenocarcinoma patients in China have an EGFR mutation. In contrast, the EGFR mutation rate was reported only $11 \%$ in US and European patients of lung adenocarcinoma [13, 14]. The clinical data from Chinese cohorts is valuable to complete the consensus guidelines and promotes FMI-guided surgery. In the following, we would like to briefly review the recent Chinese clinical achievements by using intraoperative FMI, including some new experiences in the second near-infrared wavelength window (NIR-II, 1,000 1,700 nm). We hope that this paper may help surgeons further explore the potential of FMI in the clinic and improve cancer surgical outcome.

\section{Intraoperative visualization of malignancies}

Complete resection is the expected goal of cancer surgery. Surgical margin delineation is therefore one of the most important decisions during surgery. FMI, which expands the eye inspection, shows a great potential to reveal more details of cancer. The surgical margins can be clearly visualized by FMI to improve surgical decisions. Although the cancer specificity is not ideal enough, FS, $\mathrm{MB}$, and ICG provide highly practical ways to perform high-precision surgery.

Hepatic cancer stays a severe threat to China. Nearly 50\% of the global hepatic cancer patients occur in China [15]. Many of these Chinese patients have a poor liver function and an obvious demand for precision surgery [16]. In the last decade, by using ICG, the value of intraoperative FMI has been well comprehended in hepatic surgery. The usage of ICG is recommended to be adjusted according to the patient's liver function $[17,18]$. At least 2 days prior to surgery is recommended for ICG injection to image the lesion in poorly functional liver [19]. With the aid of FMI, highdifficulty hepatic surgeries can become much safer in implementation [20-22]. For example, the highly difficult laparoscopic anatomical resection of hepatic segment VII could be successfully performed by the precise margin visualization [23]. A clear reveal of the cancer margin directly helps reach the maximum liver function preservation (Fig. 2), especially for the patients with removable hepatic recurrences [24, 25]. Another method to visualize hepatic cancer margin was using intraoperative ICG injection. The cancer area would be rapidly distinguished in a non-fluorescent manner [26]. In this study, Zhang et al. also gave the suggestions of administration routes based on their observations. A retrospective analysis conducted by Dai and colleagues demonstrated that FMI-guided resection could achieve a wider negative margin, and therefore lead to a better prognosis compared with the traditional surgery [27]. With its promising performance, FMI-guided robotic surgery may stand for a future trend of precision treatment. Li et al. have preliminarily studied the efficacy of this novel robotic surgery on 23 patients with focal nodal hyperplasia [28].

Metastasis removal is another expectation for cancer surgery. The hepatic metastasis surgery using ICG FMI has
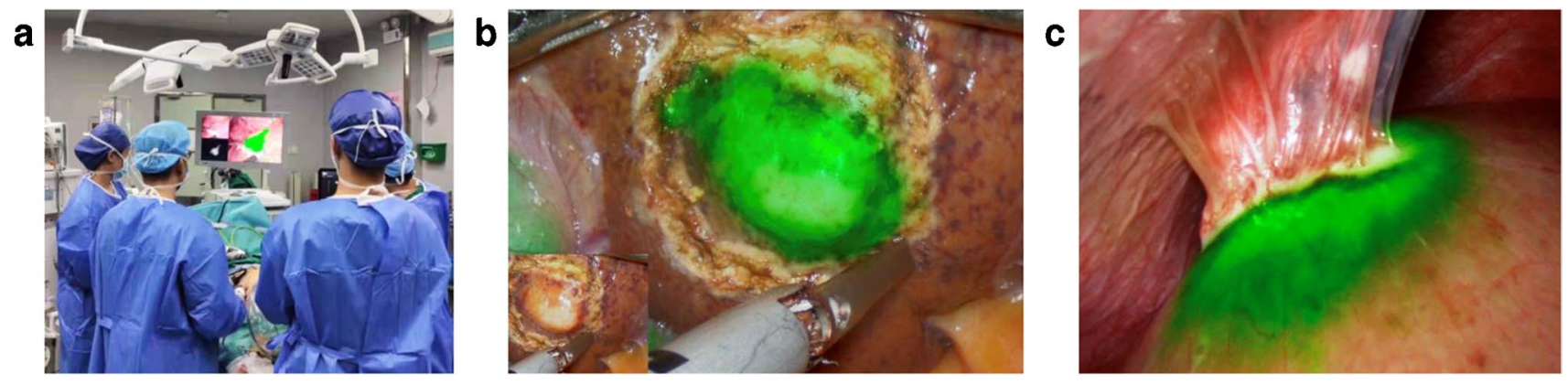

Fig. 2 Typical intraoperative FMI of malignancies. a FMI "expands" the visions of the surgeons during surgery. b Visualization of welldifferentiated HCC under the fluorescent laparoscopic view. c A liver metastasis of colorectal cancer was delineated fluorescently. The images were acquired using DPM-III-01 system (Zhuhai Dipu Medical Technology Co., Ltd.) intraoperatively 
already been included in the consensus guidelines [29]. A possible hindrance to ICG-guided hepatic metastasis surgery is the false-positive. As mentioned above, many hepatic cancer patients in China have the problem of liver cirrhosis and false-positive FMI results are usually observed. Intraoperative use of ICG may partially overcome this problem by negative-staining the cancer lesions [26], whereas the sensitivity and specificity may be affected. Recent progresses showed new FMI applications to visualize cancer in three-dimension (3D) $[30,31]$. The 3D imaging approach proposed by Fang and colleagues can help surgeons delineate the surgical area with higher accuracy. Consequently, the patients could have well-preserved liver function and shortened hospitalization. Using the 3D FMI, surgeons can assess the liver volume to be resected during surgery. High consistency between the resected volume and the surgical plan was achieved in their studies [32, 33]. Precise hepatic resection therefore could be expected to excellently preserve the functional liver volume. In China, liver function preservation is a key consideration of hepatic surgery because of the hepatitis virus infection. This practical intraoperative 3D FMI may become a widely accepted approach in the future. Another study performed by $\mathrm{He}$ et al. exhibited the merits of ICG FMI in surgery of colorectal liver metastases [34]. In their research, ICG FMI localized more lesions than the traditional inspection $(3.03 \pm 1.58$ versus $2.28 \pm 1.35, P=0.045)$. Importantly, the 1 -year cancer recurrence rate was significantly reduced by using intraoperative FMI (ICG group 19\% versus $47 \%$ in control group, $P=0.017$ ).

Pulmonary surgery is a great developing area for ICG FMI as well [35-37]. It has been verified that $24 \mathrm{~h}$ preoperative injection of $5.0 \mathrm{mg} / \mathrm{kg}$ ICG through the peripheral vein can obtain a good performance to image and resect the pulmonary nodules. The surgeries were usually conducted with thoracoscopic observation. Guided by ICG FMI, a higher precision could be achieved in pulmonary nodule detection and resection. In a study with 36 enrolled patients accomplished Tian, Wang, and colleagues, the FMI located nine occult lesions that were missed by preoperative imaging [38]. This finding is believed valuable to facilitate the surgical decisions. Their final data showed a sensitivity of $88.7 \%$ and a positive predictive value of $92.6 \%$. The surgical area could also be visualized in a negative-stained manner. Intraoperative injections of $0.2 \sim 0.6 \mathrm{mg} / \mathrm{kg}$ ICG through the peripheral venous catheter have been evaluated in different trials $[39,40]$. The surgical area would remain non-fluorescent and surrounded by the obviously fluorescent normal pulmonary tissues.

Fluorescein-guided FMI surgery has also been studied, especially for glioma surgery. Because of the disruption of blood-brain barrier (BBB), the non-specific fluorescein was able to accumulate and illuminate the glioma [41, 42]. Given that a low-dose $(1-2 \mathrm{mg} / \mathrm{kg})$ fluorescein has been proved capable of guiding the cerebral cancer surgery $[42,43]$, this BBB disruption-dependent mechanism makes this fluorescein FMI method more suitable to high-grade gliomas. The sensitivity for low-grade lesions might be lower than expected [41]. A study involving 220 patients with spinal gliomas (by Sun et al.) provided an obvious evidence that the fluorescein FMI could improve the resection rate of the MRI-enhanced lesions [44]. This intraoperative method was also proved a strong feasibility to image astrocytoma and the brain metastasis from other malignancies $[45,46]$. The potential of fluorescein FMI to raise the surgical outcomes in pediatric patients with brainstem gliomas was also explored by Xue et al. [47]. A total glioma removal was accomplished on 9 patients, while another 3 cases had a mean resection rate of $93.7 \%$. Yet we should notice that the non-specific fluorescein may cause false-negative margin to the highgrade glioma [48]. Expanded careful exploration may be needed to achieve the precision resection without residue.

Compared with ICG and fluorescein, 5-ALA and MB are used less frequently in China so far. A study (by Chan et al.) with 16 enrolled glioma patients showed that the orally taken 5 -ALA $\left(20 \mathrm{mg} / \mathrm{kg} \cdot \mathrm{m}^{2}\right)$ could specifically illuminate the glioma and aid in achieving microscopic total resection [49]. To the superficially located lesions, 5-ALA coating could be a feasible alternative to oral administration. In surgeries of penile-scrotal extramammary Paget's disease, FMI from the coated 5-ALA helped accurately delineate the surgical area [50]. Another agent, MB, is often used as a staining dye to locate the lymph nodes [51-53]. While using its fluorescent property, MB showed the feasibility to visualize the breast tumor intraoperatively; yet the effects from preoperative chemotherapy should be noticed [54].

These above trials represent the efforts made to improve surgical outcome in China. ICG applications have been widely studied, particularly in hepatic cancer. The NIR emission and the plasma-binding property also make ICG more suitable to perform in vivo cancer imaging [55]. Although the cancer specificity of these imaging agents is limited, the FMI performance could be improved by adjusting the agent administration. Moreover, combined employment of these fluorescent agents could be an effective and practical way to further amend the surgical outcome [56].

\section{Cancer imaging in NIR-II spectrum}

In 2017-2018, ICG was confirmed capable of emitting the fluorescence within the second near-infrared wavelength window (NIR-II, 1000-1700 nm) [57-59]. This important discovery expanded the application area of ICG and provided a quick way to perform NIR-II imaging in clinic.

The first-in-human NIR-II imaging study was completed in 2020 [60]. In this 23 liver cancer patients' study, Hu 
et al. verified the feasibility of intraoperative NIR-II FMI and compared the performances between NIR-II and NIR-I imaging. Notably, NIR-II imaging found 3 extra lesions that were missed by NIR-I, leading to a meaningful increase of the sensitivity (NIR-II 100\% versus NIR-I 90.63\%) and the accuracy (NIR-II 91.43\% versus NIR-I 82.86\%). This study opens the door to following clinical translations of NIR-II and multi-spectrum imaging techniques.

A NIR-II-guided glioma surgery has been reported by Shi et al. [61]. This new surgical method was performed on 41 glioma patients; the high-grade glioma detection rate was reported as $100 \%$ using ICG NIR-II imaging. Traditional white-light surgery was performed as the comparison. From their follow-up data, significant improvements can be seen in the progression-free survival (median of NIR-II group 9 months, while white-light group 7 months) and overall survival (median of NIR-II group was 19.0 months; whitelight group was 15.5 months). When integrating machine learning, more diagnostic information could be extracted from the glioma NIR-II results [62]. Shen et al. designed deep convolutional neural networks to automatically analyze the NIR-II glioma in situ images. The machine learning method had a sensitivity of $93.8 \%$ (versus $82.0 \%$ by neurosurgeons) in glioma detection, and could rapidly predict the cancer grade and even the Ki-67 expression. This interesting study exhibited the potential of machine learning for FMI and showed an intraoperative pattern to implement.

ICG NIR-II imaging has been employed as well in nephron-sparing surgery [63]. Determining the margin of renal carcinoma is a challenging problem. Cao et al. used ICG to delineate the lesion's margin by NIR-II negative fluorescence. After the injection of ICG, the normal renal parenchyma was illuminated in NIR-II imaging while the lesion area remained dark. The contrast ratio between the lesion and the normal tissue could reach over 4.8 , which was distinguishable and ideal for negative-staining imaging. Complete cancer resection was achieved in all the enrolled patients.

These recent clinical observations of NIR-II showed notable improvements on imaging performance and the surgical outcome. Benefiting from high spatial resolution, strong imaging contrast, and enhanced penetration, NIR-II with ICG showed better performance in small lesion detection and margin determination [55]. The integration of NIR-II imaging and machine learning is also attractive. A compact and economical system is beneficial to promote the use of NIR-II imaging. And multi-center involved randomized clinical trials are expected to better verify the surgical outcomes between NIR-II and traditional approaches. Briefly, more clinical applications regarding NIR-II should be encouraged in clinic.

\section{Lymphatic FMI imaging}

Dissection of lymph nodes is important for many cancer surgeries, such as breast cancer, gastric cancer, and colorectal cancer [64-66]. The main goal is to remove the possible cancer metastases in the lymphatic system [67, 68]. Locating lymph nodes is often difficult in surgery because of the complicated connection between lymphatic vessels [69]. FMI-guided lymphadenectomy can reduce the noncompliance rate and lead to a better prognosis [70, 71]. In China, the FMI-guided lymphadenectomy has been proved worth spreading in gastric cancer, which has a specifically high incurrence rate in Eastern Asia [72].

A double-armed randomized clinical trial involving 266 patients was conducted by Huang and colleagues, to study the feasibility and efficacy of ICG FMI-guided laparoscopic lymphadenectomy [73]. The patients in the experimental group received an endoscopic peritumoral injection of ICG to the submucosa 1 day before surgery. When laparoscopic D2 lymphadenectomy was performed, ICG fluorescence could retrieve $50.5 \pm 15.9$ lymph nodes, while the control group can retrieve $42.0 \pm 10.3$ nodes. Thus, the intraoperative ICG FMI was demonstrated efficient to improve gastric D2 lymphadenectomy without increased complications (Fig. 3c). The same surgical team further compared the performance of sub-mucosal and sub-serosal injection of ICG [74]. A total of 259 patients were enrolled in this per-protocol analysis. The clinical results
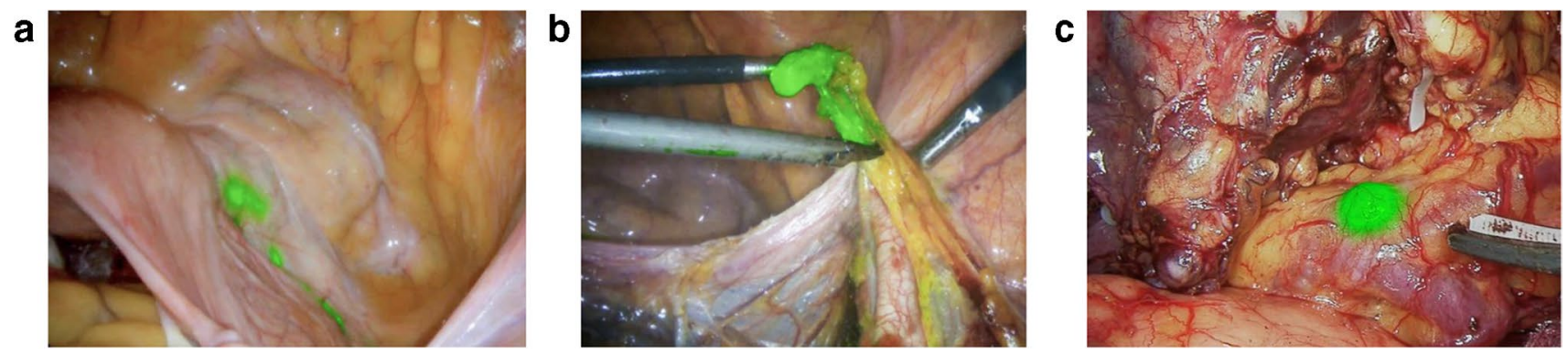

Fig. 3 FMI-guided lymphadenectomy in cervical cancer (a, b) and gastric cancer (c). The images were acquired using DPM-III-01 system (Zhuhai Dipu Medical Technology Co., Ltd.) intraoperatively 
indicated a comparable performance between sub-mucosal and sub-serosal injection (lymph node noncompliance rate 32.3\% versus $33.3 \%, P=0.860$ ). Yet the sub-mucosal method needs a preoperative gastroscopy, which may cause mental burden and extra economic cost. Therefore, the sub-serosal injection is more recommended to perform ICG FMI-guided D2 lymphadenectomy. In an analysis by Huang et al., the improved D2 lymphadenectomy method was proved valuable for advanced gastric cancer with poor outcome to neoadjuvant chemotherapy [75]. A total of 184 matched patients were included and divided into two groups equally. Clinical observation showed that the blood loss in ICG FMI group was less than the nonICG group. And the D2 dissection lymph nodes in ICG FMI group $(39.6 \pm 13.2)$ were significantly higher than the non-ICG group (30.8 \pm 11.8$)$. ICG FMI-guided D2 lymphadenectomy should be performed routinely for the patients with advanced gastric cancer. To evaluate the feasibility of imaging metastatic lymph nodes using ICG, Zhong et al. performed a 514 patients enrolled study [76]. The ICG FMI had a sensitivity of $86.8 \%$ to the metastatic lymph nodes, while the negative predictive value was $92.2 \%$ to the non-fluorescent nodes. The sensitivity and negative predictive value were also found related to the cancer stage. For cT1 c CT2 patients, both sensitivity and negative predictive value reached $100 \%$. To the cT3 cT4a patients, the sensitivity was reduced but still over $80 \%$. These findings could aid surgeons to perform personalized lymphadenectomy based on the cancer stage.

In thoracic esophageal cancer surgery, ICG FMI-guided lymphadenectomy exhibited ideal performance (by Shan and colleagues) [77]. A four-quadrant endoscopic injection was used after anesthetization. In all the 84 involved patients, the average number of regionally dissected lymph nodes was $25.68 \pm 12.00$. The stained lymph nodes showed a clear mapping for dissection, but the efficacy to identify metastasispositive nodes has not been explored yet.

These randomized clinical trials have provided high-level evidence of the FMI performance in lymphatic imaging. ICG presents a high effectiveness to visualize the regional lymph nodes and aid lymphadenectomy. However, the goal to specifically identify the metastatic nodes remains unachieved using ICG. Employing newly designed molecular-targeted imaging agents might overcome this problem [78], and multi-spectrum imaging is also believed as a feasible approach [56].

\section{Intraoperative FMI aids reduce iatrogenic injury}

Iatrogenic injury is catching attention nowadays. A series of Chinese groups have shared their experience to rduce iatrogenic injury by utilizing intraoperative FMI.

Biliary injury in hepatic surgery may cause severe complication and lead to a bad prognosis. ICG FMI showed the feasibility to visualize biliary duct intraoperatively and reduce the incurrence of biliary injury. Especially for the patients with extensive dense adhesions around the hepatoduodenal ligament, FMI can provide important assistance to avoid biliary injury [79]. Su, Li, and colleagues implemented this method through an injection of low-dose ICG $(2.5 \mathrm{mg})$ in surgical procedures. Further study also demonstrated that the biliary duct imaging performance could be optimized with $10 \mathrm{mg}$ ICG injections 10 to $12 \mathrm{~h}$ prior to surgery [80]. ICG FMI can also play a role in living donor liver transplantation [81]. By visualizing the surgical margin and the biliary branches in real time, the hepatic function of the donor could be preserved as much as possible. This method has been proved safe and feasible by $\mathrm{Li}$ et al. in pediatric liver transplantation, which guided in situ reduction of liver segment and effectively preserved liver function of the donor [82]. If more high-level evidence supported, ICG FMI may become an efficient alternative to the complicated intraoperative cholangiography in specific hepatic surgeries. Analogous preservation demand exists in thoracic surgery. The FMI method using ICG has been reported (by Yang et al.) to intraoperatively image and preserve the thoracic duct [83]. Subcutaneously injected ICG $(0.2 \mathrm{mg} / \mathrm{kg})$ into the bilateral inguinal region ( $0.5 \mathrm{~h}$ prior to surgery), any fistula sites could be clearly found and repaired with FMI observation. Similar use of ICG or fluorescein also performed a precision FMI angiography in the high-precision-demanded cephalic and eye-sparing surgery [84, 85].

The application of FMI has been expanded to the emerging nerve imaging in recent years. Most nerves are thin and indistinguishable by human eye observation [86, 87]. Complications (like palmar hyperhidrosis) are common because of the iatrogenic injury to the vulnerable nerves [88, 89]. In 2016, ICG FMI has been reported (by Wang and colleagues) as an efficient method to visualize and help preserve the nerves during thoracoscopic surgeries [90]. He et al. further validated the ICG usage on rabbits and a 15-patient cohort [91]. Their findings indicated that preoperative injection ( $24 \mathrm{~h}$ prior to surgery) of $5 \mathrm{mg} / \mathrm{kg}$ ICG could achieve an ideal performance (imaging contrast ratio $3.26 \pm 0.57$ ) of thoracic sympathetic nerve imaging. The retrospective data from 142 patients verified that the ICG nerve imaging is safe and practical to help reduce complication rates [92]. There is also a noticeable ongoing trial aiming at preserving pelvic nerves in radical hysterectomy of cervical cancer [93]. Similar ICG FMI strategy was introduced in the registration (NCT04224467, recruiting in Nanfang Hospital). Although surgical outcomes have been achieved, the mechanism of ICG intake by nerves is not crystallized yet. It is worth believing that FMI might become more effective in nerve preservation when the mechanism can be fully understood. 


\section{Multi-modal imaging promotes precision surgery}

So far in China, cancer targeted fluorescent agents are rarely reported in the clinic. Applying ICG, FS, MB, and 5-ALA has inevitable false-negative and false-positive, which may affect the surgical accuracy. The successful translations of molecular-targeting fluorescent agent like OTL38 reflect the future trend for precision surgery. But the route of developing promising molecular-targeting FMI agents is long, and strong multidisciplinary collaboration is indispensable. In the process of producing new molecular-targeting fluorescent agents, multi-modal imaging has a great worth to elevate the surgical accuracy [94, 95].

PET often represents the highest sensitivity of preoperative cancer diagnosis [96, 97]. Different from FMI, the innovative PET tracers could be translated into the clinic efficiently [98, 99]. Many cancer-specific PET tracers have been proposed in China and contributed to surgical planning $[100,101]$. The whole-body PET scan could achieve a better staging and therefore lead to precise clinical decisions [97, 102, 103]. Clinical innovations with newly designed radioactive tracers can be found in detection of hepatic cancer [100], pulmonary cancer [104, 105], glioma [106], prostate cancer [107-110], melanoma [111], lymphoma [112], etc. These new PET tracers have significantly improved the sensitivity and specificity for cancer detection. However, the impressive performance of PET can hardly be replicated in surgery. In many times, the high-quality diagnostic images may only have a limited value to surgery.

From 2009, the emerging Cerenkov luminescence imaging (CLI) provides a way to perform optical imaging using PET tracers [113, 114]. CLI makes the $\beta+$ radiotracers become dual-modal imaging agents with high specificity to cancer, and it has been proved safe to perform CLIguided surgery [115-117]. The main concern about CLI for clinical application is the low intensity of the luminescence $[118,119]$. Endoscopic CLI (ECLI) was accordingly proposed in 2012 by Cheng and colleagues [120]. The cavities of human body provide ideal environments to acquire the CLI signals. Wu, Wang, Chen, and colleagues described the first human results of ECLI [121]. Four patients with rectal cancer were involved in this study. The lesions were detected after ${ }^{18} \mathrm{~F}$-FDG injection, and the intraoperative ECLI observation is highly consistent with the preoperative PET diagnosis. Their work demonstrated the feasibility of cancer surgery guided by PET-optical dual modal molecular imaging. Nevertheless, the optical signal emitted from PET tracer is quite weak [122-124]. For ECLI, over $90 \%$ of the luminescence was measured to be attenuated during the transmission in the clinical endoscope [125]. Hence, a long exposure time was needed to obtain high-quality results, which might hinder the surgical process [126, 127]. Recently, intraoperative ex vivo CLI has become an emerging way for cancer margin detection [128-130]. The positive margins could be visualized intraoperatively by ex vivo CLI imaging. Meanwhile, multidisciplinary efforts have been done in China to discover novel methods to strengthen the performance of CLI [118, $123,124]$, whereas these new methods are still in preclinical stages. It is worth believing that the rapid upgradation of PET tracers and imaging devices could make CLI become a powerful tool for precision surgery in the future.

Another novel dual-modal imaging strategy showed the great worth of combining PET and FMI [131]. Li et al. designed a new tracer ${ }^{68}$ Ga-IRDye $800 \mathrm{CW}-\mathrm{BBN}$ targeting gastrin-releasing peptide receptor (GRPR) that is overexpressed in glioblastoma multiforme. This tracer owned the capability to perform both preoperative PET and intraoperative FMI. The results from 42 foci showed that the ${ }^{68} \mathrm{Ga}$-IRDye800CW-BBN-guided surgery had a sensitivity of $93.9 \%$ and specificity of $100 \%$. With the help of the highspecificity FMI detection, maximum safe resection is easier to achieve and $82.76 \%$ of the enrolled patients received a complete resection [132]. This PET-FMI strategy might be a valuable trial to build up the connection between molecularspecific diagnosis and surgery. Combining the results from PET and FMI, the clinical decision and the treatment outcome are believed to be hugely improved.

Furthermore, surgical observations suggest the complementation between intraoperative ultrasonography and FMI. Ultrasonography has a good performance to detect deep lesions, while FMI can visualize the surgical margin clearly and reveal small occult lesions [133]. A combination of these two intraoperative techniques could have an expanded vision and higher imaging precision [133, 134]. This combined strategy has aided to improve the $\mathrm{R} 0$ resection rate in China. Intraoperative ultrasonography can also guide ICG injection in laparoscopic surgeries for different purposes. A single-center study in 2020 shared the experiences of using intraoperative ultrasonography combing with FMI, to achieve precise hepatic segmentectomy, hemi-hepatectomy, and multi-segmentectomy [135].

\section{The coming future of precision surgery in China}

The population with newly diagnosed cancer is still growing in China. There is an urgent need of precision surgery. We can foresee that the application of intraoperative FMI would be rapidly expanded $[93,136]$. So far, the clinical value of non-specific fluorophores has been widely explored. Some 


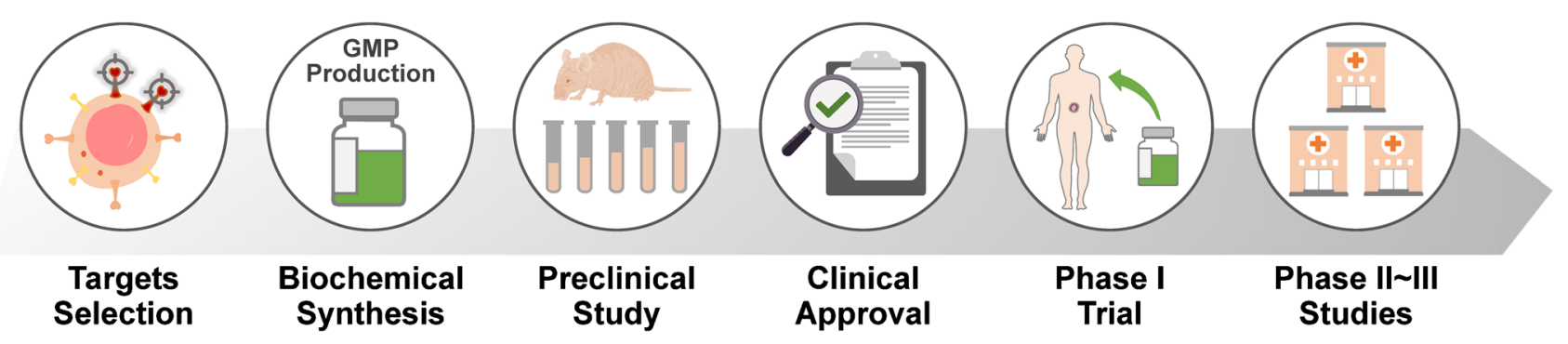

Fig. 4 The major steps of developing molecular-targeting fluorescent agents for clinical application

promising results like nerve imaging have also been studied in the clinic [93]. Nevertheless, the accuracy of malignancy imaging remains unsatisfied. Lacking molecular-targeting fluorescent agents restrains the progress of precision surgery. Analytical data showed that there have been 39 new molecular-targeting fluorescent agents reported in the clinic globally (until February 2020) [137]. Antibody-dye conjugations (e.g., bevacizumab-IRDye800CW) and specially designed small molecules (e.g., OTL38) exhibited promising performances in surgical navigation [138, 139]. Yet only few of these novel fluorescent agents were relevant with Chinese groups [137]. Comprehensive efforts from basic research to clinic are required to build up an innovative environment in China for the coming era of precision surgery. From the successful translation of the new cancer-specific fluorescent agents, the essential procedures can be summarized as follows (Fig. 4): (1) determining specific bio-targets according to cancer types, (2) synthesizing the molecular-targeting fluorescent agents in compliance with good manufacturing practice (GMP) regulation, (3) testing the developed agents with living animals, (4) applying for approval of clinical trial when the agents passed preclinical tests, (5) safety and effectiveness study in patients of a small cohort, (6) further clinical study with expanded cohorts.

As mentioned above, many cancers (e.g., pulmonary cancer) in China have a special molecular profile. The existing innovative agents from abroad may not be directly suitable for Chinese cancer patients. Thus, we strongly encourage to research new surgical solution based on the characteristics of Chinese local patients. Closer collaboration between clinicians and scientists is necessary. Participation from medicine industry is also needed. The industry of innovative medicine is growing fast, and has a bright future in China [140]. Those developing companies could provide solid foundations to study novel molecular-targeting fluorescent agents.

Another notable challenge for precision cancer surgery is the heterogeneity. The existing high-level researches have shown us the complicated cellular and molecular mechanisms during the cancer progression [141-143]. These analyses pointed out the necessities to research multi-molecular-targeting imaging. A recent study exhibited the feasibility and promising performance of FMI to intraoperatively visualize the cancer heterogeneity. Chen et al. applied multiplexed FMI to improve the diagnostic accuracy of Barrett's neoplasia [144]. A total of 22 patients were involved in this feasibility trial. The statistical analyses showed that the sensitivity and the specificity were $92 \%$ and $89 \%$, respectively. We believe that this impressive research may open the door to image multi-biomarker in surgery, while the potential of multi-modal surgical navigation should be further explored as well.

\section{Conclusion}

To reach the goal of precision surgery, a series of technical efforts have been made globally. FMI has contributed to improving various surgeries in China, and the application of NIR-II fluorescence may become a new trend. However, lacking cancer-targeted agents is still a great obstacle to achieve precision surgery. We hope the impressive progress of anti-cancer drug innovation could boost the development of molecular targeted tracers in China. A closer cooperation between clinicians and scientists is also strongly recommended to accelerate the progress towards the new era of precision surgery.

Author contribution $\mathrm{ZZ}$ and $\mathrm{KH}$ performed the literature search. CC analyzed the data. The draft was organized by ZZ, KH, and CC. ZH and JT critically revised the work. All authors read and approved the final manuscript.

Funding This study was funded by the National Key Research and Development Program of China (2017YFA0205200), National Natural Science Foundation of China (NSFC) (62027901, 92059207, 81930048, 81930053, 81227901, 82102236, 82001984, 81801864), the Chinese Academy of Sciences (QYZDJ-SSW-JSC005), Beijing Natural Science Foundation (JQ19027), Beijing Municipal Science \& Technology Commission (Z201100006820017, Z19111000110000), China Postdoctoral Science Foundation (2021M690007), the innovative research team of high-level local universities in Shanghai, and the Zhuhai High-level Health Personnel Team Project (Zhuhai HLHPTP201703). 


\section{Declarations}

Ethics approval This article does not contain any studies with human participants performed by any of the authors.

Informed consent Informed consent was obtained from all individual participants included in the study.

Conflict of interest The authors declare no competing interests.

Open Access This article is licensed under a Creative Commons Attribution 4.0 International License, which permits use, sharing, adaptation, distribution and reproduction in any medium or format, as long as you give appropriate credit to the original author(s) and the source, provide a link to the Creative Commons licence, and indicate if changes were made. The images or other third party material in this article are included in the article's Creative Commons licence, unless indicated otherwise in a credit line to the material. If material is not included in the article's Creative Commons licence and your intended use is not permitted by statutory regulation or exceeds the permitted use, you will need to obtain permission directly from the copyright holder. To view a copy of this licence, visit http://creativecommons.org/licenses/by/4.0/.

\section{References}

1. Mieog JSD, Achterberg FB, Zlitni A, Hutteman M, Burggraaf J, Swijnenburg R-J, et al. Fundamentals and developments in fluorescence-guided cancer surgery. Nat Rev Clin Oncol. 2021;1:14.

2. Lauwerends LJ, van Driel PBAA, Baatenburg de Jong RJ, Hardillo JAU, Koljenovic S, Puppels G, et al. Real-time fluorescence imaging in intraoperative decision making for cancer surgery. Lancet Oncol. 2021;22:e186-95.

3. Tipirneni KE, Warram JM, Moore LS, Prince AC, de Boer E, Jani AH, et al. Oncologic procedures amenable to fluorescenceguided surgery. Ann Surg. 2017;266:36-47.

4. Chi C, Du Y, Ye J, Kou D, Qiu J, Wang J, et al. Intraoperative imaging-guided cancer surgery: from current fluorescence molecular imaging methods to future multi-modality imaging technology. Theranostics. 2014;4:1072-84.

5. Hernot S, van Manen L, Debie P, Mieog JSD, Vahrmeijer AL. Latest developments in molecular tracers for fluorescence imageguided cancer surgery. Lancet Oncol. 2019;20:e354-67.

6. Quan YH, Oh CH, Jung D, Lim J-Y, Choi BH, Rho J, et al. Evaluation of intraoperative near-infrared fluorescence visualization of the lung tumor margin with indocyanine green inhalation. JAMA Surg American Medical Association. 2020;155:732-40.

7. Lwin TM, Hoffman RM, Bouvet M. The future of tumour-specific fluorescence-guided surgery for pancreatic cancer. Lancet Gastroenterol Hepatol. 2020;5:715-7.

8. Zhang RR, Schroeder AB, Grudzinski JJ, Rosenthal EL, Warram JM, Pinchuk AN, et al. Beyond the margins: real-time detection of cancer using targeted fluorophores. Nat Rev Clin Oncol. 2017;14:347-64.

9. van Dam GM, Themelis G, Crane LMA, Harlaar NJ, Pleijhuis RG, Kelder W, et al. Intraoperative tumor-specific fluorescence imaging in ovarian cancer by folate receptor- $\alpha$ targeting: first in-human results. Nat Med. 2011;17:2472.

10. Tipirneni KE, Rosenthal EL, Moore LS, Haskins AD, Udayakumar N, Jani AH, et al. Fluorescence imaging for cancer screening and surveillance. Mol Imaging Biol. 2017;19:645-55.
11. Achterberg FB, Deken MM, Meijer RPJ, Mieog JSD, Burggraaf $\mathrm{J}$, van de Velde $\mathrm{CJH}$, et al. Clinical translation and implementation of optical imaging agents for precision image-guided cancer surgery. Eur J Nucl Med Mol Imaging. 2021;48:332-9.

12. FDA approves new imaging drug to help identify ovarian cancer lesions. FDA. 2021 November 29. Available from: https://www. fda.gov/news-events/press-announcements/fda-approves-newimaging-drug-help-identify-ovarian-cancer-lesions

13. Wang R, Pan Y, Li C, Zhang H, Garfield D, Li Y, et al. Analysis of major known driver mutations and prognosis in resected adenosquamous lung carcinomas. J Thorac Oncol Off Publ Int Assoc Study Lung Cancer. 2014;9:760-8.

14. Cheng T-YD, Cramb SM, Baade PD, Youlden DR, Nwogu C, Reid ME. The international epidemiology of lung cancer: latest trends, disparities, and tumor characteristics. J Thorac Oncol Off Publ Int Assoc Study Lung Cancer. 2016;11(1653):71.

15. Sung H, Ferlay J, Siegel RL, Laversanne M, Soerjomataram I, Jemal A, et al. Global cancer statistics 2020: GLOBOCAN estimates of incidence and mortality worldwide for 36 cancers in 185 countries. CA Cancer J Clin. 2021;71:209-49.

16. Singal AG, Lampertico $P$, Nahon P. Epidemiology and surveillance for hepatocellular carcinoma: new trends. J Hepatol Elsevier. 2020;72:250-61.

17. Wang Q, Li X, Qian B, Hu K, Liu B. Fluorescence imaging in the surgical management of liver cancers: current status and future perspectives. Asian J Surg. 2021;S1015-9584(21):00567-74.

18. Zhang W, Hu Z, Tian J, Fang C. A narrative review of nearinfrared fluorescence imaging in hepatectomy for hepatocellular carcinoma. Ann Transl Med. 2021;9:171.

19. Digital MAOCMA, Digital ISPCOCRH, Liver CPCOCMDA, Clinical PMPC, Medical IAEPCOCGS, Molecular IPCOCBS. Guidelines for application of computer-assisted indocyanine green molecular fluorescence imaging in diagnosis and surgical navigation of liver tumors (2019). Nan Fang Yi Ke Da Xue Xue Bao. 2019;39:1127-40.

20. Li J, Li X, Zhang X, Wang H, Li K, He Y, et al. Indocyanine green fluorescence imaging-guided laparoscopic right posterior hepatectomy. Surg Endosc. 2021;36(2):1293-301.

21. Yang J, Tao H-S, Luo W, Chen R, Lin J-Y, Zhu W, et al. A novel method of fluorescent imaging can guide hepatectomy for intrahepatic cholangiocarcinoma with intrahepatic biliary obstruction. J Surg Oncol. 2020;122:1580-6.

22. He K, Hong X, Chi C, Cai C, Wang K, Li P, et al. A new method of near-infrared fluorescence image-guided hepatectomy for patients with hepatolithiasis: a randomized controlled trial. Surg Endosc. 2020;34:4975-82.

23. He J, Zhen Z, Ye Q, Mo J, Chen G, Peng J. Laparoscopic anatomical segment VII resection for hepatocellular carcinoma using the Glissonian approach with indocyanine green dye fluorescence. J Gastrointest Surg. 2020;24:1228-9.

24. Li B, Liu T, Zhang Y, Zhang J. Retroperitoneal laparoscopic hepatectomy of recurrent hepatocellular carcinoma: case report and literature review. BMC Gastroenterol. 2020;20:278.

25. Ishizawa T, Masuda K, Urano Y, Kawaguchi Y, Satou S, Kaneko $\mathrm{J}$, et al. Mechanistic background and clinical applications of indocyanine green fluorescence imaging of hepatocellular carcinoma. Ann Surg Oncol. 2014;21:440-8.

26. Zhang Y-M, Shi R, Hou J-C, Liu Z-R, Cui Z-L, Li Y, et al. Liver tumor boundaries identified intraoperatively using real-time indocyanine green fluorescence imaging. J Cancer Res Clin Oncol. 2017;143:51-8.

27. $\mathrm{Lu} \mathrm{H}, \mathrm{Gu} J$, Qian X-F, Dai X-Z. Indocyanine green fluorescence navigation in laparoscopic hepatectomy: a retrospective singlecenter study of 120 cases. Surg Today. 2021;51:695-702. 
28. Li C-G, Zhou Z-P, Tan X-L, Wang Z-Z, Liu Q, Zhao Z-M. Robotic resection of liver focal nodal hyperplasia guided by indocyanine green fluorescence imaging: a preliminary analysis of 23 cases. World J Gastrointest Oncol. 2020;12:1407-15.

29. Wang X, Teh CSC, Ishizawa T, Aoki T, Cavallucci D, Lee S-Y, et al. Consensus guidelines for the use of fluorescence imaging in hepatobiliary surgery. Ann Surg. 2021;274:97-106.

30. Zhang W, Zhu W, Yang J, Xiang N, Zeng N, Hu H, et al. Augmented reality navigation for stereoscopic laparoscopic anatomical hepatectomy of primary liver cancer: preliminary experience. Front Oncol. 2021;11:663236.

31. Zhang P, Luo H, Zhu W, Yang J, Zeng N, Fan Y, et al. Real-time navigation for laparoscopic hepatectomy using image fusion of preoperative 3D surgical plan and intraoperative indocyanine green fluorescence imaging. Surg Endosc. 2020;34:3449-59.

32. Yang J, Tao H-S, Cai W, Zhu W, Zhao D, Hu H-Y, et al. Accuracy of actual resected liver volume in anatomical liver resections guided by 3-dimensional parenchymal staining using fusion indocyanine green fluorescence imaging. J Surg Oncol. 2018;118:1081-7.

33. Zheng J, Feng X, Cai J, Tao L, Liang X. Laparoscopic anatomical portal territory hepatectomy with cirrhosis by Takasaki's approach and indocyanine green fluorescence navigation (with video). Ann Surg Oncol. 2020;27:5179-80.

34. He K, Hong X, Chi C, Cai C, An Y, Li P, et al. Efficacy of nearinfrared fluorescence-guided hepatectomy for the detection of colorectal liver metastases: a randomized controlled trial. J Am Coll Surg. 2022;234(2):130-7.

35. Zhou J, Yang F, Jiang G, Wang J. Applications of indocyanine green based near-infrared fluorescence imaging in thoracic surgery. J Thorac Dis. 2016;8:S738-43.

36. Newton AD, Predina JD, Nie S, Low PS, Singhal S. Intraoperative fluorescence imaging in thoracic surgery. J Surg Oncol. 2018;118:344-55.

37. Matsuura Y, Ichinose J, Nakao M, Okumura S, Mun M. Recent fluorescence imaging technology applications of indocyanine green in general thoracic surgery. Surg Today. 2020;50:1332-42.

38. Mao Y, Chi C, Yang F, Zhou J, He K, Li H, et al. The identification of sub-centimetre nodules by near-infrared fluorescence thoracoscopic systems in pulmonary resection surgeries. Eur $\mathbf{J}$ Cardiothorac Surg. 2017;52:1190-6.

39. Li H, Zhou J, Chi C, Mao Y, Yang F, Tian J, et al. Clinical application of near-infrared thoracoscope with indocyanine green in video-assisted thoracoscopic bullectomy. $\mathbf{J}$ Thorac Dis. 2016;8:1841-5.

40. Li H, Zhou J, Yang F, Wang J. Identifying interlobar fissure in a Craig grade 4 fissureless patient by near-infrared thoracoscopy. J Thorac Dis. 2018;10:E52-4.

41. Xiang Y, Zhu X-P, Zhao J-N, Huang G-H, Tang J-H, Chen $\mathrm{H}-\mathrm{R}$, et al. Blood-brain barrier disruption, sodium fluorescein, and fluorescence-guided surgery of gliomas. Br J Neurosurg. 2018;32:141-8.

42. Fan C, Jiang Y, Liu R, Wu G, Wu G, Xu K, et al. Safety and feasibility of low-dose fluorescein-guided resection of glioblastoma. Clin Neurol Neurosurg. 2018;175:57-60.

43. Lin F-H, Zhang X-H, Zhang J, He Z-Q, Duan H, Ke C, et al. Fluorescein sodium-guided biopsy or resection in primary central nervous system lymphomas with contrast-enhancing lesion in MRI. J Neurooncol. 2018;139:757-65.

44. Sun Z, Jing L, Fan Y, Zhang H, Chen L, Wang G, et al. Fluorescein-guided surgery for spinal gliomas: analysis of 220 consecutive cases. Int Rev Neurobiol. 2020;151:139-54.

45. Xiao S-Y, Zhang J, Zhu Z-Q, Li Y-P, Zhong W-Y, Chen J-B, et al. Application of fluorescein sodium in breast cancer brainmetastasis surgery. Cancer Manag Res. 2018;10:4325-31.
46. Zhang J, Al-Nahari F, Wang Z-F, Lin F-H, Zhao Y-Y, Xiao S-Y, et al. Application of fluorescein sodium in the resection of vermis pilocytic astrocytomas. World J Surg Oncol. 2017; 15:46.

47. Xue Z, Kong L, Pan C-C, Wu Z, Zhang J-T, Zhang L-W. Fluorescein-guided surgery for pediatric brainstem gliomas: preliminary study and technical notes. J Neurol Surg Part B Skull Base. 2018;79:S340-6.

48. Chen Z-B, Zhu X-P, Zheng W, Xiang Y, Huang Y-K, Fang $\mathrm{H}-\mathrm{J}$, et al. Relationship between the sodium fluorescein yellow fluorescence boundary and the actual boundary of high-grade gliomas during surgical resection. Br J Neurosurg. 2021;1:8.

49. Chan DTM, Yi-Pin Sonia H, Poon WS. 5-Aminolevulinic acid fluorescence guided resection of malignant glioma: Hong Kong experience. Asian J Surg. 2018;41:467-72.

50. Peng X, Qian W, Hou J. 5-aminolevulinic acid (5-ALA) fluorescence-guided Mohs surgery resection of penile-scrotal extramammary Paget's disease. Biosci Trends. 2017;11:595-9.

51. Rozenholc A, Samouelian V, Warkus T, Gauthier P, Provencher $\mathrm{D}$, Sauthier P, et al. Green versus blue: randomized controlled trial comparing indocyanine green with methylene blue for sentinel lymph node detection in endometrial cancer. Gynecol Oncol. 2019;153:500-4.

52. Agrawal SK, Hashlamoun I, Karki B, Sharma A, Arun I, Ahmed R. Diagnostic performance of indocyanine green plus methylene blue versus radioisotope plus methylene blue dye method for sentinel lymph node biopsy in node-negative early breast cancer. JCO Glob Oncol. 2020;6:1225-31.

53. Guo J, Yang H, Wang S, Cao Y, Liu M, Xie F, et al. Comparison of sentinel lymph node biopsy guided by indocyanine green, blue dye, and their combination in breast cancer patients: a prospective cohort study. World J Surg Oncol. 2017;15:196.

54. Zhang C, Jiang D, Huang B, Wang C, Zhao L, Xie X, et al. Methylene blue-based near-infrared fluorescence imaging for breast cancer visualization in resected human tissues. Technol Cancer Res Treat. 2019;18:1533033819894331.

55. Hu Z, Chen W-H, Tian J, Cheng Z. NIRF nanoprobes for cancer molecular imaging: approaching clinic. Trends Mol Med. 2020;26:469-82.

56. Blair S, Garcia M, Davis T, Zhu Z, Liang Z, Konopka C, et al. Hexachromatic bioinspired camera for image-guided cancer surgery. Sci Transl Med. 2021;13:eaaw7067.

57. Starosolski Z, Bhavane R, Ghaghada KB, Vasudevan SA, Kaay A. Annapragada A. Indocyanine green fluorescence in second near-infrared (NIR-II) window. Plos One. 2017;12:e0187563.

58. Zhu S, Hu Z, Tian R, Yung BC, Yang Q, Zhao S, et al. Repurposing cyanine NIR-I dyes accelerates clinical translation of nearinfrared-II (NIR-II) bioimaging. Adv Mater. 2018;9:e1802546.

59. Carr JA, Franke D, Caram JR, Perkinson CF, Saif M, Askoxylakis V, et al. Shortwave infrared fluorescence imaging with the clinically approved near-infrared dye indocyanine green. Proc Natl Acad Sci. 2018;115:4465-70.

60. Hu Z, Fang C, Li B, Zhang Z, Cao C, Cai M, et al. First-in-human liver-tumour surgery guided by multispectral fluorescence imaging in the visible and near-infrared-I/II windows. Nat Biomed Eng. 2020;4(3):259-71.

61. Shi X, Zhang Z, Zhang Z, Cao C, Cheng Z, Hu Z, et al. Nearinfrared window II fluorescence image-guided surgery of highgrade gliomas prolongs the progression-free survival of patients. IEEE Trans Biomed Eng. 2021. https://doi.org/10.1109/TBME. 2021.3130195 .

62. Shen B, Zhang Z, Shi X, Cao C, Zhang Z, Hu Z, et al. Real-time intraoperative glioma diagnosis using fluorescence imaging and deep convolutional neural networks. Eur J Nucl Med Mol Imaging. 2021;48:3482-92.

63. Cao C, Deng S, Wang B, Shi X, Ge L, Qiu M, et al. Intraoperative near-infrared II window fluorescence imaging-assisted 
nephron-sparing surgery for complete resection of cystic renal masses. Clin Transl Med. 2021;11:e604.

64. Huang Y, Liang C, He L, Tian J, Liang C, Chen X, et al. Development and validation of a radiomics nomogram for preoperative prediction of lymph node metastasis in colorectal cancer. J Clin Oncol. 2016;34:2157-64.

65. Kim MJ, Park SC, Park JW, Chang HJ, Kim DY, Nam B-H, et al. Robot-assisted versus laparoscopic surgery for rectal cancer: a phase II open label prospective randomized controlled trial. Ann Surg. 2018;267:243-51.

66. Xia C, Zhou Q, Zhang Q, Hu S, Meacci E, Matsuura Y, et al. Comparative study on the diagnostic value of intravenous/peritumoral injection of indocyanine green for metastatic lymph node location in patients with head and neck squamous cell carcinoma (HNSCC). Ann Transl Med. 2021;9:507.

67. Okubo K, Uenosono Y, Arigami T, Matsushita D, Yanagita S, Kijima T, et al. Quantitative assessment of fluorescence intensity of ICG in sentinel nodes in early gastric cancer. Gastric Cancer. 2018;21:776-81.

68. Zhang C, Li Y, Wang X, Zhang M, Jiang W, Ou J. Clinical study of combined application of indocyanine green and methylene blue for sentinel lymph node biopsy in breast cancer. Medicine (Baltimore). 2021;100:e25365.

69. Wang Y, Zhu L, Xia W, Wang F. Anatomy of lymphatic drainage of the esophagus and lymph node metastasis of thoracic esophageal cancer. Cancer Manag Res. 2018;10:6295-303.

70. Roh CK, Choi S, Seo WJ, Cho M, Son T, Kim H-I, et al. Indocyanine green fluorescence lymphography during gastrectomy after initial endoscopic submucosal dissection for early gastric cancer. Br J Surg. 2020;107:712-9.

71. Zapardiel I, Alvarez J, Barahona M, Barri P, Boldo A, Bresco P, et al. Utility of intraoperative fluorescence imaging in gynecologic surgery: systematic review and consensus statement. Ann Surg Oncol. 2021;28:3266-78.

72. Rahman R, Asombang AW, Ibdah JA. Characteristics of gastric cancer in Asia. World J Gastroenterol. 2014;20:4483-90.

73. Chen Q-Y, Xie J-W, Zhong Q, Wang J-B, Lin J-X, Lu J, et al. Safety and efficacy of indocyanine green tracer-guided lymph node dissection during laparoscopic radical gastrectomy in patients with gastric cancer: a randomized clinical trial. JAMA Surg. 2020;155:300-11.

74. Chen Q-Y, Zhong Q, Li P, Xie J-W, Liu Z-Y, Huang X-B, et al. Comparison of submucosal and subserosal approaches toward optimized indocyanine green tracer-guided laparoscopic lymphadenectomy for patients with gastric cancer (FUGES-019): a randomized controlled trial. BMC Med. 2021;19:276.

75. Huang Z-N, Yan S, Qiu WW, Liu CH, Chen QY, Zheng CH, et al. Assessment of indocyanine green tracer-guided lymphadenectomy in laparoscopic gastrectomy after neoadjuvant chemotherapy for locally advanced gastric cancer: results from a multicenter analysis based on propensity matching. Gastric Cancer Off J Int Gastric Cancer Assoc Jpn Gastric Cancer Assoc. 2021;24(1355):64.

76. Zhong Q, Chen Q-Y, Huang X-B, Lin G-T, Liu Z-Y, Chen J-Y, et al. Clinical implications of indocyanine green fluorescence imaging-guided laparoscopic lymphadenectomy for patients with gastric cancer: a cohort study from two randomized, controlled trials using individual patient data. Int J Surg Lond Engl. 2021;94:106120.

77. Wang X, Hu Y, Wu X, Liang M, Hu Z, Gan X, et al. Near-infrared fluorescence imaging-guided lymphatic mapping in thoracic esophageal cancer surgery. Surg Endosc. 2021. https://doi.org/ 10.1007/s00464-021-08720-7.

78. Lu G, van den Berg NS, Martin BA, Nishio N, Hart ZP, van Keulen S, et al. Tumour-specific fluorescence-guided surgery for pancreatic cancer using panitumumab-IRDye800CW: a phase 1 single-centre, open-label, single-arm, dose-escalation study. Lancet Gastroenterol Hepatol. 2020;5:753-64.

79. Wang P, Fang C, Lin H-M, Li X-J, Luo D, Gan Y, et al. Detection of the common bile duct in difficult bile duct exploration using indocyanine green fluorescence imaging: a case report. Photodiagnosis Photodyn Ther. 2021;36:102610.

80. Chen Q, Zhou R, Weng J, Lai Y, Liu H, Kuang J, et al. Extrahepatic biliary tract visualization using near-infrared fluorescence imaging with indocyanine green: optimization of dose and dosing time. Surg Endosc. 2021;35:5573-82.

81. Meng X, Wang H, Xu Y, Chen M, Duan W, Lu S. Indocyanine green fluorescence image-guided total laparoscopic living donor right hepatectomy: the first case report from Mainland China. Int J Surg Case Rep. 2018;53:406-9.

82. Li H, Zhu Z, Wei L, Tan Y, Zeng Z, Qu W, et al. Laparoscopic left lateral monosegmentectomy in pediatric living donor liver transplantation using real-time ICG fluorescence in situ reduction. J Gastrointest Surg. 2020;24:2185-6.

83. Yang F, Zhou J, Li H, Yang F, Xiao R, Chi C, et al. Near-infrared fluorescence-guided thoracoscopic surgical intervention for postoperative chylothorax. Interact Cardiovasc Thorac Surg. 2018;26:171-5.

84. Ye Z-P, Yang X-Y, Li W-S, Hou B, Guo Y. Microsurgical resection of cervical spinal cord arteriovenous malformations: report of 6 cases. World Neurosurg. 2016;96:362-9.

85. Zhou N, Xu X, Wei W. Analysis of clinical features of RPE adenoma. Graefes Arch Clin Exp Ophthalmol. 2020;258:2831-40.

86. Liu J, Pan L, Shang C, Lu B, Wu R, Feng Y, et al. A highly sensitive and selective nanosensor for near-infrared potassium imaging. Sci Adv. 2020;6(16):eaax9757.

87. Wang LG, Barth CW, Kitts CH, Mebrat MD, Montaño AR, House BJ, et al. Near-infrared nerve-binding fluorophores for buried nerve tissue imaging. Sci Transl Med. 2020;12:eaay0712.

88. Monje M, Borniger JC, D'Silva NJ, Deneen B, Dirks PB, Fattahi $\mathrm{F}$, et al. Roadmap for the emerging field of cancer neuroscience. Cell. 2020;181:219-22.

89. Walsh EM, Cole D, Tipirneni KE, Bland KI, Udayakumar N, Kasten BB, et al. Fluorescence imaging of nerves during surgery. Ann Surg. 2019;270:69-76.

90. Weng W, Liu Y, Zhou J, Li H, Yang F, Jiang G, et al. Thoracoscopic indocyanine green near-infrared fluorescence for thoracic sympathetic ganglions. Ann Thorac Surg Elsevier. 2016;101:2394.

91. He K, Zhou J, Yang F, Chi C, Li H, Mao Y, et al. Near-infrared intraoperative imaging of thoracic sympathetic nerves: from preclinical study to clinical trial. Theranostics. 2018;8:304-13.

92. Pei G, Liu Y, Liu Q, Min X, Yang Y, Wang S, et al. The safety and feasibility of intraoperative near-infrared fluorescence imaging with indocyanine green in thoracoscopic sympathectomy for primary palmar hyperhidrosis. Thorac Cancer. 2020;11:943-9.

93. He K, Li P, Zhang Z, Liu J, Liu P, Gong S, et al. Intraoperative near-infrared fluorescence imaging can identify pelvic nerves in patients with cervical cancer in real time during radical hysterectomy. Eur J Nucl Med Mol Imaging. 2022. https://doi.org/10. 1007/s00259-022-05686-Z.

94. Wang S, Zhao Y, Xu Y. Recent advances in applications of multimodal ultrasound-guided photoacoustic imaging technology. Vis Comput Ind Biomed Art. 2020;3(1):24.

95. An Y, Wang K, Tian J. Recent methodology advances in fluorescence molecular tomography. Vis Comput Ind Biomed Art. 2018;1(1):1.

96. Kang F, Han Q, Zhou X, Zheng Z, Wang S, Ma W, et al. Performance of the PET vascular activity score (PETVAS) for qualitative and quantitative assessment of inflammatory activity 
in Takayasu's arteritis patients. Eur J Nucl Med Mol Imaging. 2020;47:3107-17.

97. Chen H, Pang Y, Wu J, Zhao L, Hao B, Wu J, et al. Comparison of [68Ga]Ga-DOTA-FAPI-04 and [18F] FDG PET/CT for the diagnosis of primary and metastatic lesions in patients with various types of cancer. Eur J Nucl Med Mol Imaging. 2020;47:1820-32.

98. Li X, Rosenkrans ZT, Wang J, Cai W. PET imaging of macrophages in cardiovascular diseases. Am J Transl Res. 2020;12:1491-514.

99. Sun X, Xiao Z, Chen G, Han Z, Liu Y, Zhang C, et al. A PET imaging approach for determining EGFR mutation status for improved lung cancer patient management. Sci Transl Med. 2018; 10:eaan8840.

100. Guo W, Pang Y, Yao L, Zhao L, Fan C, Ke J, et al. Imaging fibroblast activation protein in liver cancer: a single-center post hoc retrospective analysis to compare [68Ga]Ga-FAPI-04 PET/ CT versus MRI and [18F]-FDG PET/CT. Eur J Nucl Med Mol Imaging. 2021;48:1604-17.

101. Long T, Hou J, Yang N, Zhou M, Li Y, Li J, et al. Utility of 18F-AlF-NOTA-octreotide PET/CT in the localization of tumor-induced osteomalacia. J Clin Endocrinol Metab. 2021;106:e4202-9.

102. Luo Y, Pan Q, Yang H, Peng L, Zhang W, Li F. Fibroblast activation protein-targeted PET/CT with 68Ga-FAPI for imaging IgG4-related disease: comparison to 18F-FDG PET/CT. J Nucl Med. 2021;62(2):266-71.

103. Ding J, Zhang Y, Wen J, Zhang H, Wang H, Luo Y, et al. Imaging CXCR4 expression in patients with suspected primary hyperaldosteronism. Eur J Nucl Med Mol Imaging. 2020;47:2656-65.

104. Kang F, Wang Z, Li G, Wang S, Liu D, Zhang M, et al. Interheterogeneity and intra-heterogeneity of $\alpha v \beta 3$ in non-small cell lung cancer and small cell lung cancer patients as revealed by $68 \mathrm{Ga}-\mathrm{RGD} 2$ PET imaging. Eur J Nucl Med Mol Imaging. 2017;44:1520-8.

105. Kang F, Mu W, Gong J, Wang S, Li G, Li G, et al. Integrating manual diagnosis into radiomics for reducing the false positive rate of 18F-FDG PET/CT diagnosis in patients with suspected lung cancer. Eur J Nucl Med Mol Imaging. 2019;46:2770-9.

106. Zhang J, Tian Y, Li D, Niu G, Lang L, Li F, et al. 68Ga-NOTAAca-BBN(7-14) PET imaging of GRPR in children with optic pathway glioma. Eur J Nucl Med Mol Imaging. 2019;46:2152-62.

107. Zhang J, Shao S, Wu P, Liu D, Yang B, Han D, et al. Diagnostic performance of 68Ga-PSMA PET/CT in the detection of prostate cancer prior to initial biopsy: comparison with cancer-predicting nomograms. Eur J Nucl Med Mol Imaging. 2019;46:908-20.

108. Liu C, Liu T, Zhang Z, Zhang N, Du P, Yang Y, et al. 68GaPSMA PET/CT combined with PET/ultrasound-guided prostate biopsy can diagnose clinically significant prostate cancer in men with previous negative biopsy results. J Nucl Med. 2020;61(9):1314-9.

109. Liu C, Liu T, Zhang N, Liu Y, Li N, Du P, et al. 68Ga-PSMA-617 PET/CT: a promising new technique for predicting risk stratification and metastatic risk of prostate cancer patients. Eur J Nucl Med Mol Imaging. 2018;45:1852-61.

110. Wang B, Liu C, Wei Y, Meng J, Zhang Y, Gan H, et al. A prospective trial of 68Ga-PSMA and 18F-FDG PET/CT in nonmetastatic prostate cancer patients with an early PSA progression during castration. Clin Cancer Res Off J Am Assoc Cancer Res. 2020;26:4551-8.

111. Ma X, Wang S, Wang S, Liu D, Zhao X, Chen H, et al. Biodistribution, radiation dosimetry, and clinical application of a melanin-targeted PET probe, 18F-P3BZA, in Patients. J Nucl Med. 2019;60(1):16-22.
112. Zhang X, Chen L, Jiang H, He X, Feng L, Ni M, et al. A novel analytic approach for outcome prediction in diffuse large B-cell lymphoma by [18F]FDG PET/CT. Eur J Nucl Med Mol Imaging. 2021. https://doi.org/10.1007/s00259-021-05572-0.

113. Zhang Z, Cai M, Bao C, Hu Z, Tian J. Endoscopic Cerenkov luminescence imaging and image-guided tumor resection on hepatocellular carcinoma-bearing mouse models. Nanomed Nanotechnol. 2019;17:62-70.

114. Zhang Z, Cai M, Gao Y, Shi X, Zhang X, Hu Z, et al. A novel Cerenkov luminescence tomography approach using multilayer fully connected neural network. Phys Med Biol. 2019;64:245010.

115. Thorek DLJ, Riedl CC, Grimm J. Clinical Cerenkov luminescence imaging of 18F-FDG. J Nucl Med. 2014;55:95-8.

116. Lee HJ, Ehlerding EB, Jiang D, Barnhart TE, Cao T, Wei W, et al. Dual-labeled pertuzumab for multimodality image-guided ovarian tumor resection. Am J Cancer Res. 2019;9:1454-68.

117. Li M, Wei W, Barnhart TE, Jiang D, Cao T, Fan K, et al. ImmunoPET/NIRF/Cerenkov multimodality imaging of ICAM-1 in pancreatic ductal adenocarcinoma. Eur J Nucl Med Mol Imaging. 2021;48:2737-48.

118. Shi X, Cao C, Zhang Z, Tian J, Hu Z. Radiopharmaceutical and Eu3+ doped gadolinium oxide nanoparticles mediated tripleexcited fluorescence imaging and image-guided surgery. J Nanobiotechnology. 2021;19:212.

119. Yu B, Ni D, Rosenkrans ZT, Barnhart TE, Wei H, Ferreira CA, et al. A "missile-detonation" strategy to precisely supply and efficiently amplify Cerenkov radiation energy for cancer theranostics. Adv Mater. 2019;31:e1904894.

120. Liu H, Carpenter CM, Jiang H, Pratx G, Sun C, Buchin MP, et al. Intraoperative imaging of tumors using Cerenkov luminescence endoscopy: a feasibility experimental study. J Nucl Med. 2012;53:1579-84.

121. Hu H, Cao X, Kang F, Wang M, Lin Y, Liu M, et al. Feasibility study of novel endoscopic Cerenkov luminescence imaging system in detecting and quantifying gastrointestinal disease: first human results. Eur Radiol. 2015;25:1814-22.

122. Hu Z, Qu Y, Wang K, Zhang X, Zha J, Song T, et al. In vivo nanoparticle-mediated radiopharmaceutical-excited fluorescence molecular imaging. Nat Commun. 2015;6:7560.

123. Hu Z, Zhao M, Qu Y, Zhang X, Zhang M, Liu M, et al. In vivo 3-dimensional radiopharmaceutical-excited fluorescence tomography. J Nucl Med. 2017;58(1):169-74.

124. Zhang Z, Qu Y, Zhang X, Guo H, Shi X, Cai M, et al. Radiopharmaceuticals and fluorescein sodium mediated triple-modality molecular imaging allows precise image-guided tumor surgery. Adv Sci. 2019;6(13):1900159.

125. Cao X, Chen X, Kang F, Lin Y, Liu M, Hu H, et al. Performance evaluation of endoscopic Cerenkov luminescence imaging system: in vitro and pseudotumor studies. Biomed Opt Express. 2014;5:3660-70.

126. Zhang Z, Qu Y, Cao Y, Shi X, Guo H, Zhang X, et al. A novel in vivo Cerenkov luminescence image-guided surgery on primary and metastatic colorectal cancer. J Biophotonics. 2019;13(3):e201960152.

127. Hu Z, Chi C, Liu M, Guo H, Zhang Z, Zeng C, et al. Nanoparticle-mediated radiopharmaceutical-excited fluorescence molecular imaging allows precise image-guided tumor-removal surgery. Nanomed Nanotechnol. 2017;13(4):1323-31.

128. Grootendorst MR, Cariati M, Pinder S, Kothari A, Douek M, Kovacs T, et al. Intraoperative assessment of tumor resection margins in breast-conserving surgery using $18 \mathrm{~F}-\mathrm{FDG}$ Cerenkov luminescence imaging - a first-in-human feasibility study. J Nucl Med. 2017;58(6):891-8.

129. oldeHeuvel J, de Wit-van der Veen BJ, van der Poel HG, Bekers EM, Grootendorst MR, Vyas KN, et al. 68Ga-PSMA Cerenkov 
luminescence imaging in primary prostate cancer: first-in-man series. Eur J Nucl Med Mol Imaging. 2020;47(11):2624-32.

130. Hu Z, Zhang Z, Guo H, Tian J. Intraoperative Cerenkov luminescence imaging for image-guided resection of low rectal cancer on swine models. J Nucl Med. 2018;59(S1):246.

131. Li D, Zhang J, Chi C, Xiao X, Wang J, Lang L, et al. First-inhuman study of PET and optical dual-modality image-guided surgery in glioblastoma using 68Ga-IRDye800CW-BBN. Theranostics. 2018;8:2508-20.

132. He K, Chi C, Li D, Zhang J, Niu G, Lv F, et al. Resection and survival data from a clinical trial of glioblastoma multiformespecific IRDye800-BBN fluorescence-guided surgery. Bioeng Transl Med. 2021;6:e10182.

133. Liu Y, Gao B, Fang C, Su S, Yang X, Tian J, et al. Application of near-infrared fluorescence imaging technology in liver cancer surgery. Surg Innov. 2021;26:1553350621997777.

134. Liu B, Liu T, Su M, Ma Y-Q, Zhang B-F, Wang Y-F, et al. Improving the surgical effect for primary liver cancer with intraoperative fluorescence navigation compared with intraoperative ultrasound. Med Sci Monit Int Med J Exp Clin Res. 2019;25:3406-16.

135. Xu Y, Chen M, Meng X, Lu P, Wang X, Zhang W, et al. Laparoscopic anatomical liver resection guided by real-time indocyanine green fluorescence imaging: experience and lessons learned from the initial series in a single center. Surg Endosc. 2020;34:4683-91.

136. Shang W, Peng L, He K, Guo P, Deng H, Liu Y, et al. A clinical study of a CD44v6-targeted fluorescent agent for the detection of non-muscle invasive bladder cancer. Eur J Nucl Med Mol Imaging. 2022. https://doi.org/10.1007/s00259-022-05701-3.
137. Barth CW, Gibbs SL. Fluorescence image-guided surgery - a perspective on contrast agent development. Proc SPIE Int Soc Opt Eng. 2020;11222:112220J.

138. Kennedy GT, Azari FS, Bernstein E, Marfatia I, Din A, Kucharczuk JC, et al. Targeted intraoperative molecular imaging for localizing nonpalpable tumors and quantifying resection margin distances. JAMA Surg. 2021;156(11):1043-50.

139. Lamberts LE, Koch M, de Jong JS, Adams ALL, Glatz J, Kranendonk MEG, et al. Tumor-specific uptake of fluorescent bevacizumab-IRDye800CW microdosing in patients with primary breast cancer: a phase I feasibility study. Clin Cancer Res. 2017;23:2730-41.

140. Li G, Qin Y, Xie C, Wu Y-L, Chen X. Trends in oncology drug innovation in China. Nat Rev Drug Discov. 2021;20:15-6.

141. Hanahan D, Weinberg RA. Hallmarks of cancer: the next generation. Cell. 2011;144:646-74.

142. Dagogo-Jack I, Shaw AT. Tumour heterogeneity and resistance to cancer therapies. Nat Rev Clin Oncol. 2018;15:81-94.

143. Heinrich S, Craig AJ, Ma L, Heinrich B, Greten TF, Wang XW. Understanding tumour cell heterogeneity and its implication for immunotherapy in liver cancer using single-cell analysis. J Hepatol. 2021;74:700-15.

144. Chen J, Jiang Y, Chang T-S, Joshi B, Zhou J, Rubenstein JH, et al. Multiplexed endoscopic imaging of Barrett's neoplasia using targeted fluorescent heptapeptides in a phase 1 proof-ofconcept study. Gut. 2021;70:1010-3.

Publisher's note Springer Nature remains neutral with regard to jurisdictional claims in published maps and institutional affiliations. 